\title{
In Vivo Human Hair Hydration Measurements by Using Opto-Thermal Radiometry
}

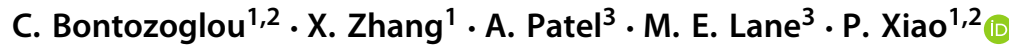

Received: 25 September 2017 / Accepted: 24 December 2018 / Published online: 28 January 2019

(c) The Author(s) 2019

\begin{abstract}
The water content in human hair is very important for its cosmetic properties and general health. However, to measure water content in hair, especially in vivo hair, is very difficult. Opto-thermal transient emission radiometry (OTTER) is a promising, infrared remote sensing technology that can be used for this kind of measurements. It can not only measure the water content but also the water depth profile in hair. By measuring the water content and its depth profile in hair, we can understand the status of hair, i.e., healthy or damaged, etc. In this paper, we will present our latest study on in vivo human hair hydration measurements by using OTTER. We will first present the theoretical background and then show the experimental results. We will also compare the OTTER hair results with other established measurement technique results, such as condenser trans-epidermal water loss method.
\end{abstract}

Keywords AquaFlux · Desorption · Hair hydration · Opto-thermal radiometry · Shampooing

\section{Introduction}

Human hair is a skin appendix that covers many parts of a human body. Hair grows from the hair follicle which can be found within the skin $[1,2]$. The hair follicle is the only living portion of the hair. The shape of the hair follicle determines the shape of hair. The Asian hair tends to be straight and has an almost round cross section. The

\footnotetext{
This article is part of the selected papers presented at the 19th International Conference on Photoacoustic and Photothermal Phenomena.

$凶 \quad$ P. Xiao

xiaop@1sbu.ac.uk

1 School of Engineering, London South Bank University, 103 Borough Road, London SE1 0AA, UK

2 Biox Systems Ltd, 90 London Road, London SE1 6LN, UK

3 UCL School of Pharmacy, 29-39 Brunswick Square, London WC1N 1AX, UK
} 

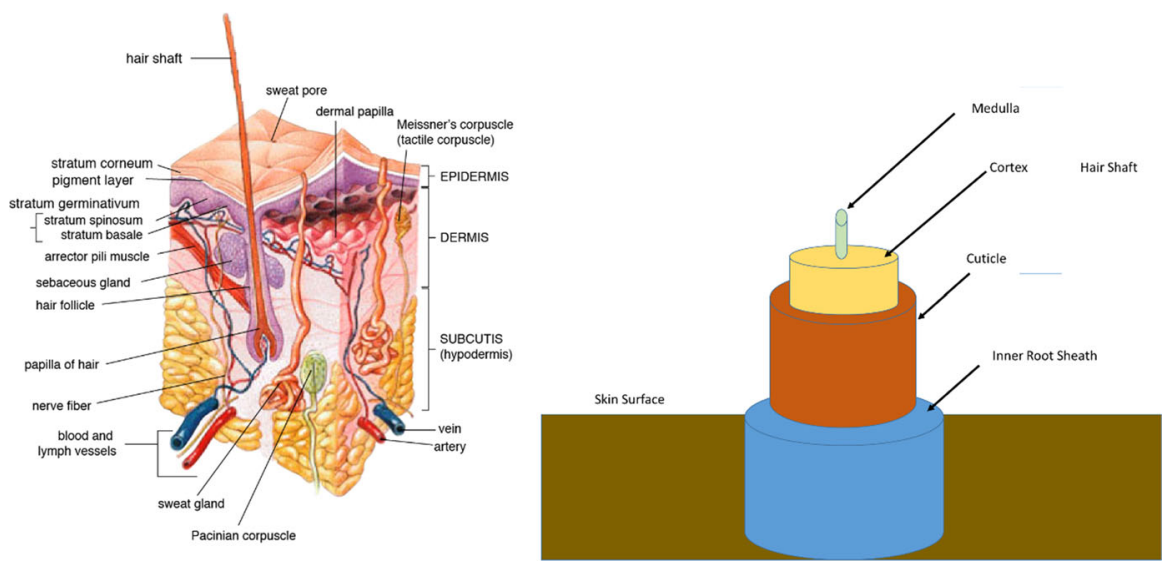

Fig. 1 The structure of the human skin and the human hair (left) [1] and the structure of the human hair shaft (right)

African hair tends to be finer and curlier and has more flattened cross section. The Caucasian hair tends to be more variable in both cross section shape and curvature. The visible part of the hair, i.e., above the skin, is called the hair shaft, which is made of dead protein filament. The root of a hair, or bulb of hair, contains the cells that produce the hair shaft. Different types of hair are produced by different kinds of follicles. Figure 1 shows the structure of human skin and human hair (left) [1], and the structure of the human hair shaft (right).

The hair contains three main layers: medulla, cortex and cuticle. The medulla is the innermost layer of the hair. It is made of soft keratin and is unstructured. The medulla only exists in large thick hairs, like the hairs on scalp rather than from the abdomen or upper arms. If exists, the medulla exists all the way along the hair. The cortex is the middle layer of hair and is made of hard keratin which gives the hair its strength. The cortex contains pigments that give the hair colors; it is also the main source for water absorption. The cortex is the thickest hair layer. Therefore, the cortex determines the hair's color, curl pattern, thickness and texture. The cuticle is the outmost layer of hair. It looks very similar to the layout of tiles on a house roof. When the hair swells, the cuticle's complex structure slides. It is covered by a layer of lipids that makes the hair repels water. There are sweat glands that can produces sweats to cool down the body, and sebaceous gland that can produce oil to lubricate the hair.

The hair on the top of the head, i.e., scalps, presents the most cosmetic value and therefore is mostly researched on. Kristensen et al. [3] have studied the effect of hair dyeing and hair washing on hair cortisol concentrations. Hamel et al. [4] have studied the effect of hair shampooing and hair washing on hair cortisol concentrations. Boll et al. [5] have studied of dyed and non-dyed hairs using ATR FT-IR spectroscopy. Barba et al. [6, 7] have also studied the water content, water absorption/desorption of hair. Water content in the hair is very important for the hair's health and cosmetic properties; however, to measure water content in the hair, especially in vivo hair, is very difficult. There are several studies using near infrared technologies to study the water content in hair, including in vivo hair [8-10]. But to date, there is no technologies 
can resolve water depth distribution in hair. Our previous studies show that it is also possible to measure the water content as well as the water depth profiles in hair by using Opto-thermal transient emission radiometry (OTTER) [11-14], and to measure desorption of hair by using condenser trans-epidermal water loss (TEWL) method [15-17]. In this paper, we will present in vivo human hair hydration measurements by using OTTER. We will first present the theoretical background and then show the experimental results. We will also compare the OTTER hair results with other established measurement technique results, such as condenser-TEWL method and DVS (dynamic vapor sorption) method.

\section{Methodology and Materials}

The apparatus used in this study is conventional Opto-thermal transient emission radiometry (OTTER) and condenser-TEWL method. OTTER is an infrared remote sensing technology, a form of pulsed photo-thermal radiometry (PTR) or opto-thermal radiometry (OTR) [10], see Fig. 2. OTTER uses a pulsed laser (Er:YAG laser, $2.94 \mu \mathrm{m}$, $100 \mathrm{~ns}$ pulse duration, $1 \mathrm{~mm}$ diameter laser spot size, a few mille joules per pulse) as a heat source to heat the sample, and a fast infrared MCT detector (mercury cadmium telluride, with FOV $=60^{\circ}$, Infrared Associates Inc, USA) to measure the sample's consequent blackbody radiation increase. From OTTER signals, we can get information of the sample, such as optical properties, thermal properties, and layered structure. The selection of detection wavelength is achieved by using narrow bandpass mid-infrared interference filters. By selecting different detection wavelengths, we can measure different properties of the sample, for example, the water concentration information in skin $(13.1 \mu \mathrm{m})$ or solvent concentration information within skin $(9.5 \mu \mathrm{m})$. The OTTER detection depth is about $20 \mu \mathrm{m}$. The diameter of human hair is in the order of one tenth of a millimeter, i.e., $100 \mu \mathrm{m}$, hair cuticle is about a few microns, so OTTER measurements are completely confined within the diameter of human hair, but should cover both hair cuticle and hair cortex.

The OTTER signal can be generally expressed as [11-14],

$$
S(t)=A e^{t / \tau} \operatorname{erfc} \sqrt{t / \tau}
$$

where $A$ is the amplitude of the signal, $\tau=\frac{1}{\beta^{2} \mathrm{D}}$ is the signal decay lifetime, $\beta$ is the sample's emission absorption coefficient, and D is the sample's thermal diffusivity. By fitting the OTTER signal using Eq. 1, we can get the best fit $\beta$, and from $\beta$ we can get the water content $\mathrm{H}$ in the sample, i.e., skin, hair, or nail [13].

$$
\mathrm{H}=\frac{\beta_{w}-\beta}{\beta_{w}-\beta_{d}}
$$

where $\beta_{\mathrm{w}}$ is the emission absorption coefficient of water, $\beta_{\mathrm{d}}$ is the emission absorption coefficient of dry sample. By using segmented least square (SLS) fitting, we can also get the water content at different depths; details are available elsewhere [14]. 


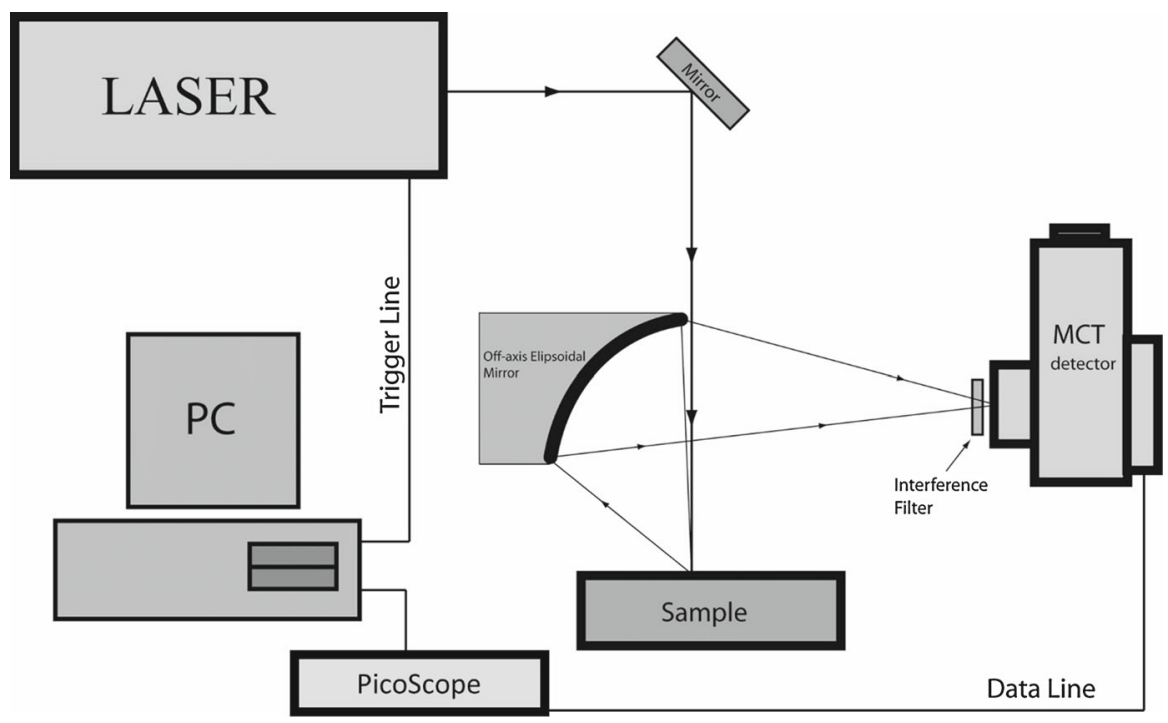

Fig. 2 The schematic diagram of OTTER measurements

The condenser-TEWL method (AquaFlux, Biox Systems Ltd, London UK) is a novel technology for TEWL (trans-epidermal water loss) measurement [11]. It is based on a closed cylindrical chamber sealed with an electronically cooled condenser $\left(-7^{\circ} \mathrm{C}\right)$. Water vapor from the skin surface will enter the measurement chamber at one end and will be frozen to ice on the condenser at the other end. This creates a steady water vapor density distribution within the measurement chamber and a water vapor density gradient. We can then calculate the flux density from the water vapor density gradient according to Fick's first law of diffusion.

$$
\mathrm{J}=-D \frac{d C}{d z}
$$

where $\mathrm{J}=$ flux density $\left(\mathrm{kg} \cdot \mathrm{m}^{2} \cdot \mathrm{s}^{-1}\right), \mathrm{D}=$ diffusion coefficient $\left(\mathrm{m}^{2} \cdot \mathrm{s}^{-1}\right), \mathrm{C}=$ concentration $\left(\mathrm{kg} \cdot \mathrm{m}^{-3}\right), \mathrm{z}=$ distance normal to the skin surface $(\mathrm{m}) . \mathrm{dC} / \mathrm{dz}$ is the water vapor density gradient. The closed chamber with a condenser makes it sure that every measurement is performed under the same condition, without the influence of external environment. As a result, AquaFlux has better repeatability and accuracy than any other TEWL instruments [12].

All the hair samples used in this study are freshly cut from healthy volunteers, prior to the measurements. The each hair samples is made of a bundle of hairs, about $2 \mathrm{~cm}$ long, about $100 \mathrm{mg}$ in weight, and wrapped at one end with aluminum foil. The hair sample was placed in a sealed cap, which is then coupled on the probe. The flux density values were then recorded for a period of $30 \mathrm{~min}$, the normalized flux density curves are then compared between different hair samples. The details of the hair desorption measurement using Condenser-TEWL method can be found in [15]. The laboratory ambient condition is $21^{\circ} \mathrm{C} \pm 2{ }^{\circ} \mathrm{C}$ and $40 \% \pm 5 \%$ relative humidity (RH). 

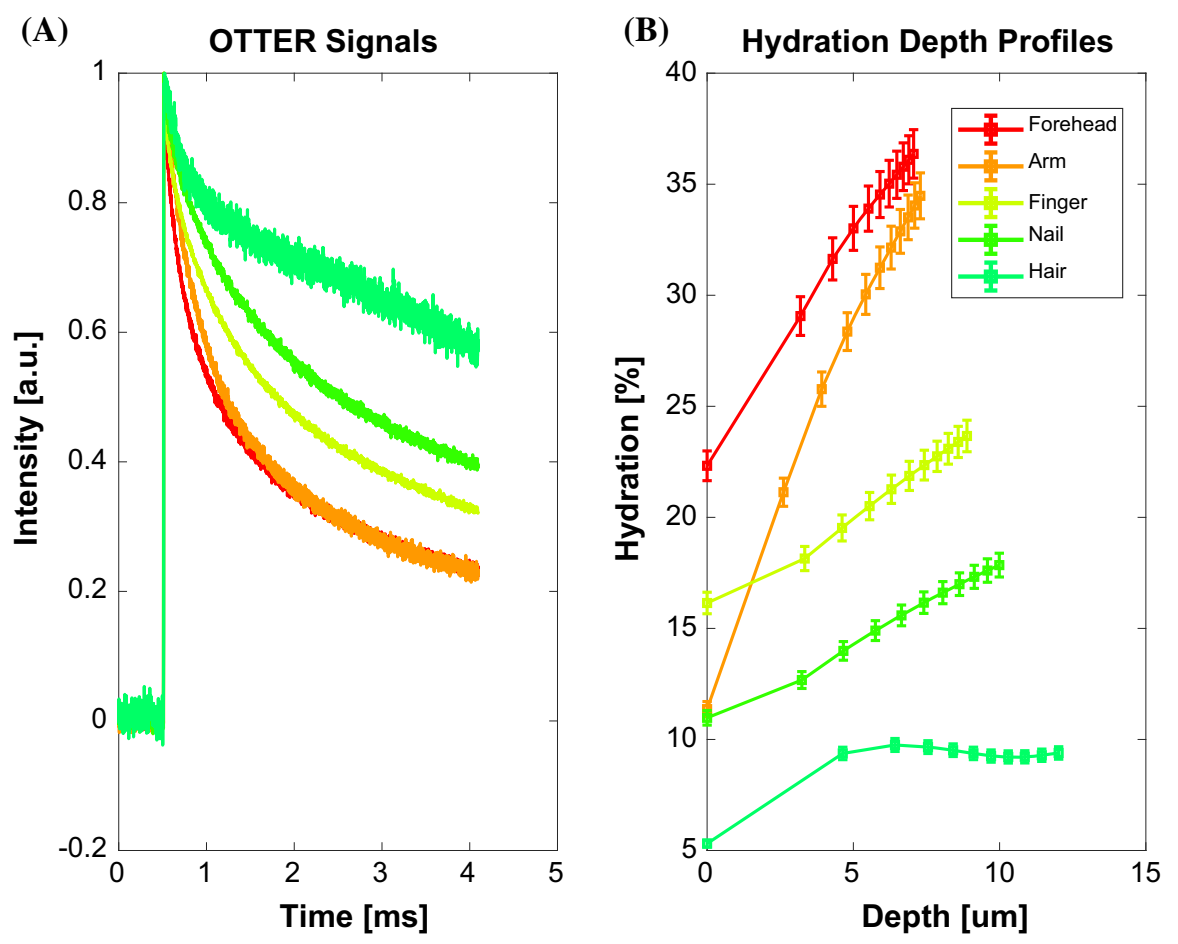

Fig. 3 The OTTER signals (a) and analyzed results (b) for the in vivo human hair, nail and skin samples

\section{Results and Discussions}

\subsection{Hair, Nail and Skin Sites}

Figure 3 shows the typical OTTER signals and corresponding hydration depth profiles for human hair, nail and in vivo skin samples. The hydration depth profiles results are calculated by using SLS fitting of the OTTER signals. The skin at the forehead shows the highest water content, and skin at the arm shows the steepest gradient. The water content in finger skin is low, and the water contents in nail and hair are even lower. The error bars show the variabilities of the measurements. Our studies show that OTTER measurements are very repeatable, with only about $3 \%$ errors. The results show that OTTER can measure not only the water content in human hair, but also the water distribution at different depth within hair, i.e., depth profiling. The water distribution in hair is significantly different from nail and skin. The curvature of the water distribution in hair is likely due to the layered structure of hair, as OTTER can measure both cuticle layer and cortex layer of the hair. No other techniques can measure the water concentration depth profiles of the top several microns of the in vivo skin with such accuracy. 

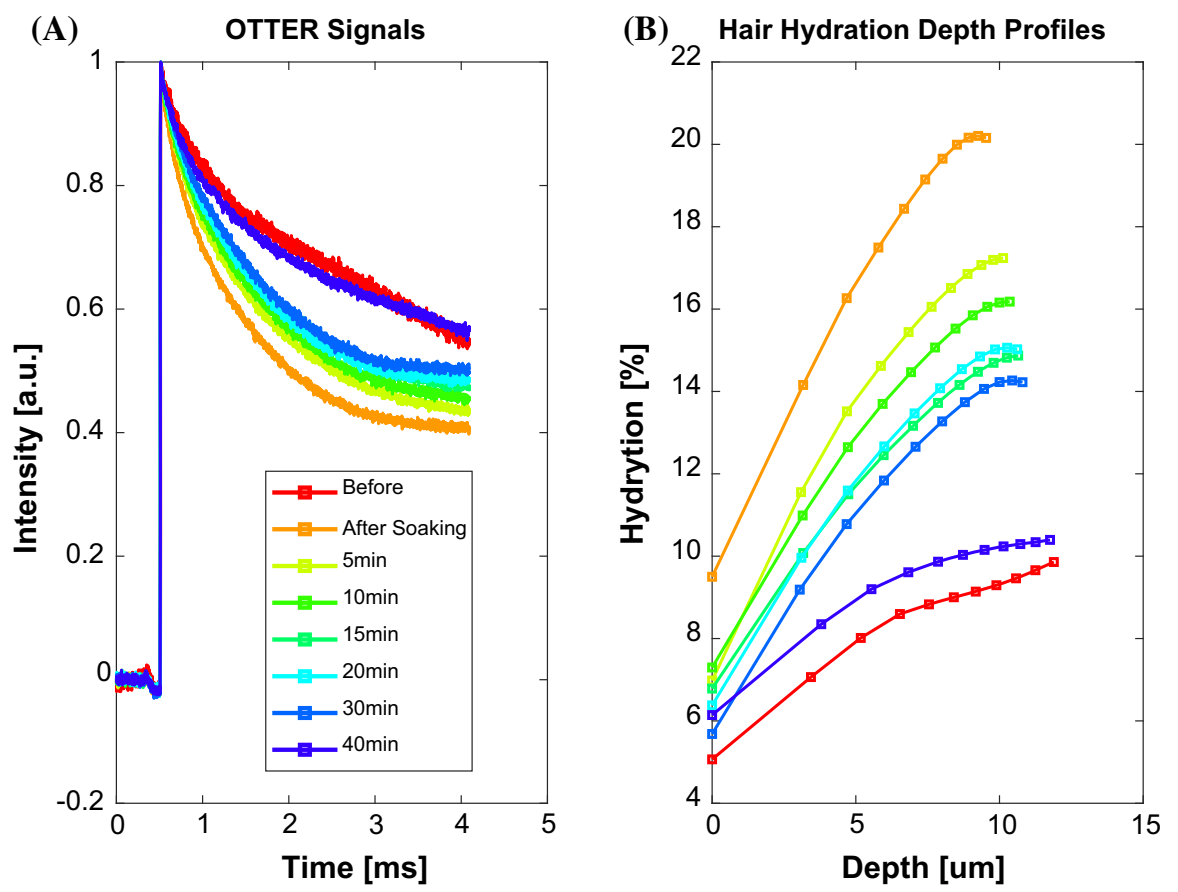

Fig. 4 The OTTER signals (a) and analyzed results (b) for in vivo human hair before and after soaking

\subsection{Hair Hydration}

Figure 4 shows the OTTER signals and the corresponding hydration depth profiles of the hair before and after the 10 min soaking in room temperature tap water. The hair sample was carefully pad-dried using a soft tissue to make sure there is no surface water present. The results show that after soaking, the hydration inside hair is significantly increased, and even more interestingly, the hair hydration depth profile seems to have lost its curvature after soaking. This suggests when the water content in hair changes, the water distribution also changes, and water distributions within hair tend to be more uniform after the hair absorbs more water from soaking. Then as the hair recovered under ambient condition, the hydration level gradually reduces to its normal level. When the hair loss its water, in the first $30 \mathrm{~min}$, the slope of the water concentration depth profiles maintains more or less the same. This suggests that the extra water in the hair is likely to be free water, and therefore they can loss independent of depth. The results show it needs more than $40 \mathrm{~min}$ to recover to its normal level.

Figure 5 shows the OTTER signals and the corresponding hydration depth profiles of the hair before and after the $10 \mathrm{~min}$ shampoo application. The results show that after shampooing, the hair hydration has also increased, and it takes more than $50 \mathrm{~min}$ to recover to its normal level.

Figure 6 shows the OTTER signals and the corresponding hydration depth profiles of the hair before and after straightening (ghd hair straightener, $180{ }^{\circ} \mathrm{C}$ and $30 \mathrm{~s}$ ). The 

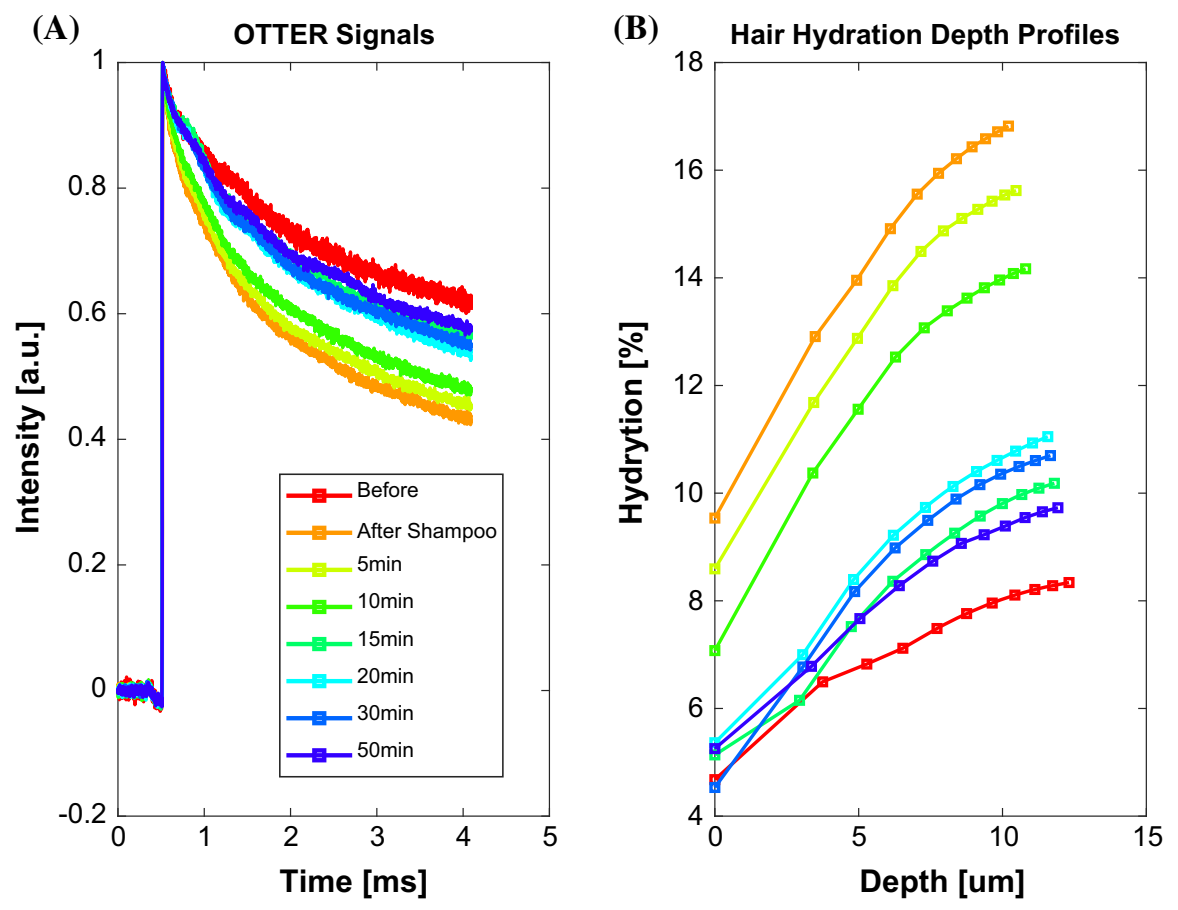

Fig. 5 The OTTER signals (a) and analyzed results (b) for in vivo human hair before and after shampooing

results show that after straightening, the hair hydration has significantly reduced. In this case, not only the water content changed, but also the water depth profile also changed, which became more flat. This is probably because the heat that the hair straightener produces breaks down the hair's hydrogen bonds and disulfide bonds, which then allows the keratin chains to move around slightly. When the hair cools down, the disulfide bonds between the keratin are reformed. The heat hence changes the hair's structure, and the hair also looks visibly straighter as well.

By plotting the hair hydration at different time, we can also study the differences of different hair samples. Figure 7 shows the hair hydration of two different hair samples (hair sample 1: Asian, black color, 13-18 years old, hair sample 2: Asian, black color, 40-50 years old) at different times before and after soaking. The hair sample 1 apparently absorbed more water during soaking and loose water much more slowly during recovering in general (except for the period between $25 \mathrm{~min}$ and $40 \mathrm{~min}$ ) than the hair sample 2. This suggests that hair sample 1, the younger hair, can hold water better than the hair sample 2, the older hair. This result generally agrees well with other measurement technologies, such desorption measurements using condenser-TEWL method and the standard DVS (dynamic vapor sorption) method, see Fig. 8. For the condenser-TEWL method, the water vapor flux density is recorded over a period of time; this reflects the water coming out of hair, i.e., desorption. For the DVS method, the weight of the hair sample is recorded over a period of time. By normalized curves, we can compare the hair samples irrespective of their quantity. The results also show 

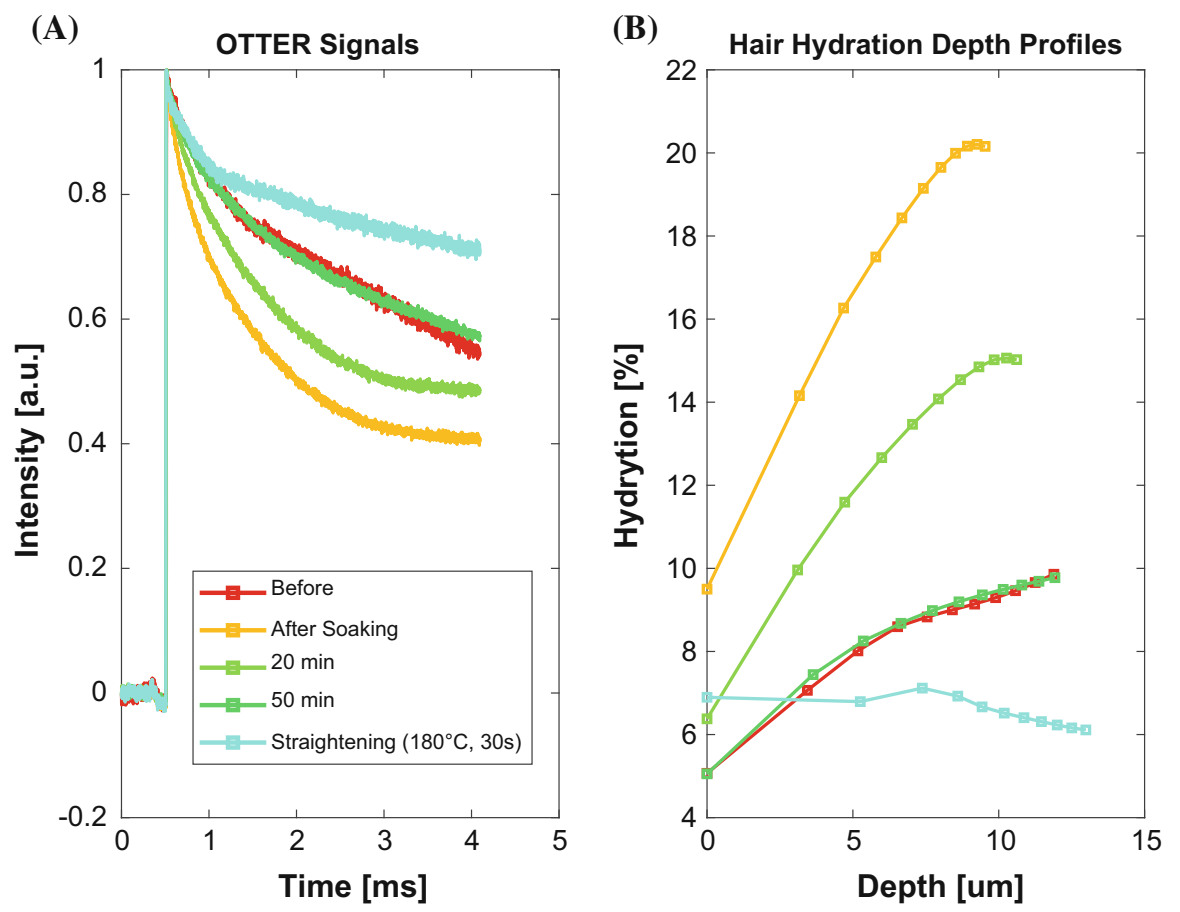

Fig. 6 The OTTER signals (a) and analyzed results (b) for in vivo human hair before and after straightening

that the hair sample 1 looses water much more slowly than the hair sample 2. So with OTTER, we can study the water holding capability of hair. Although we used hair samples in the paper, OTTER is fully capable of measuring the in vivo hair, i.e., without cutting it off.

Figure 9 shows how the hair hydration changes differently after soaking and shampooing. The results show that for the same hair sample, the hair absorbs more water during soaking than during shampooing, and the hair also loose water more slowly. This is very interesting, as potentially we can use OTTER to study the effect of different shampoos on different types of hairs.

\section{Conclusions}

The results show that opto-thermal transient emission radiometry (OTTER) is a promising technology that can be used for in vivo hair hydration measurements. OTTER can measure not only the water content in hair but also its distributions at different depth, i.e., depth profiles. The OTTER depth profiling results show that the water distribution within hair is not uniform. The curvature of the water distribution in hair is likely due to the layered structure of hair, as OTTER can measure both cuticle layer and cortex layer of the hair. The results also show that OTTER can effectively measure the hair hydration changes due to soaking, shampooing and straightening. 
Hair Hydration - Soaking

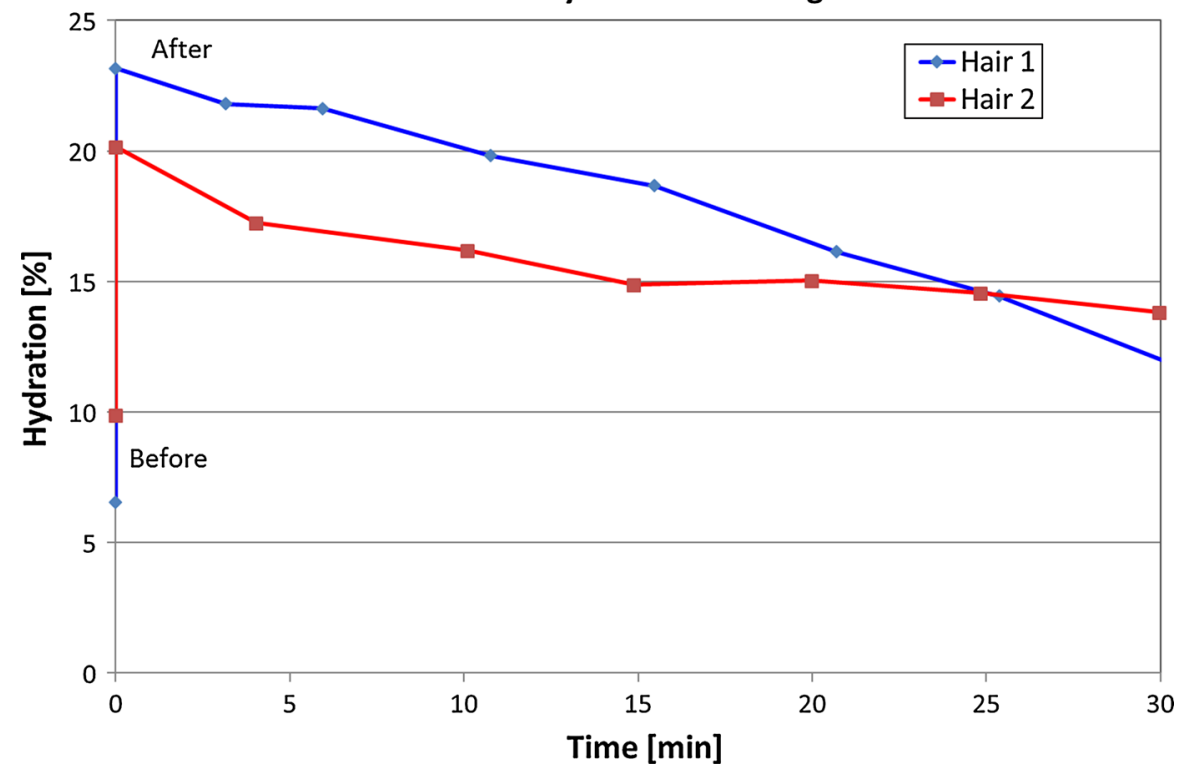

Fig. 7 The hair hydration of two different hair samples at different times before and after soaking

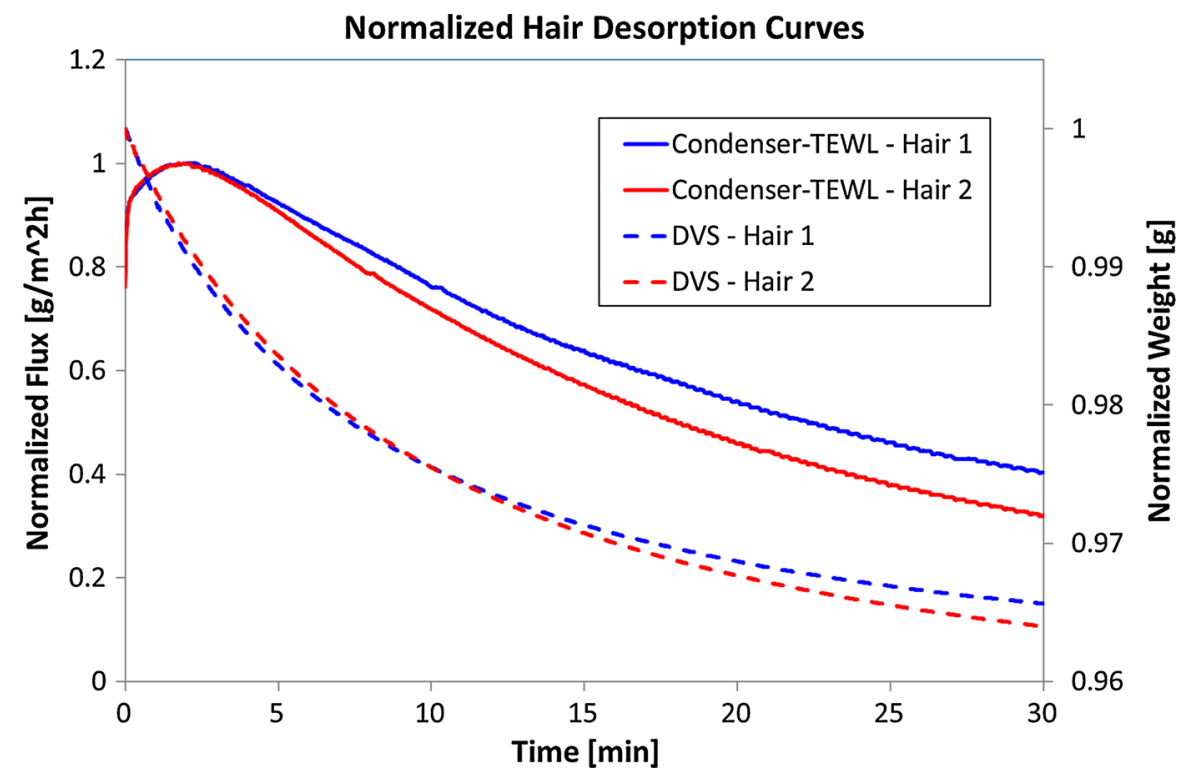

Fig. 8 The hair desorption results of the same two hair samples as shown in Fig. 7, by using the CondenserTEWL method (left Y axis) and by using the DVS (dynamic vapor sorption) method (right Y axis)

OTTER can also measure the differences of different hair samples. In the cases of hair soaking and hair straightening, the water depth profiles in the hair are also changed. 


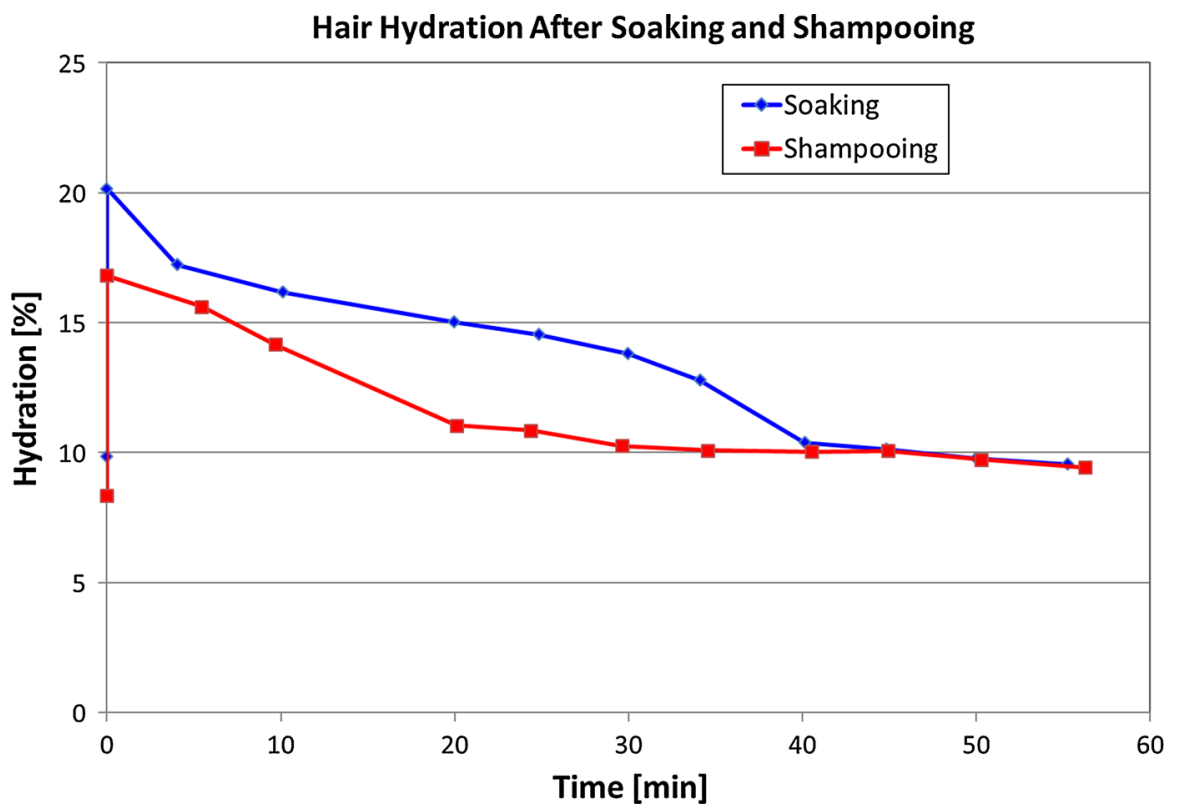

Fig. 9 The hair hydration changes of one hair sample at different times after soaking and shampooing

Finally, the OTTER results generally agree well with other measurement technique, such as hair desorption measurements using condenser-TEWL method and the standard DVS (dynamic vapor sorption) method.

Acknowledgements We thank London South Bank University for the financial support. We also thank Biox Systems Ltd for the studentship support for C. Bontozoglou.

Open Access This article is distributed under the terms of the Creative Commons Attribution 4.0 International License (http://creativecommons.org/licenses/by/4.0/), which permits unrestricted use, distribution, and reproduction in any medium, provided you give appropriate credit to the original author(s) and the source, provide a link to the Creative Commons license, and indicate if changes were made.

\section{References}

1. https://en.wikipedia.org/wiki/Human_skin. Accessed 21 Sept 2017

2. https://en.wikipedia.org/wiki/Hair. Accessed 21 Sept 2017

3. S.K. Kristensen, S.C. Larsen, N.J. Olsen, J. Fahrenkrug, B.L. Heitmann, Hair dyeing, hair washing and hair cortisol concentrations among women from the healthy start study. Psychoneuroendocrinology 77, 182-185 (2017). https://doi.org/10.1016/j.psyneuen.2016.12.016

4. A.F. Hamel, J.S. Meyer, E. Henchey, A.M. Dettmer, S.J. Suomi, M.A. Novak, Effects of shampoo and water washing on hair cortisol concentrations. Clin. Chim. Acta 412, 382-385 (2011). https://doi.org/ 10.1016/j.cca.2010.10.019

5. M.S. Boll, K.C. Doty, R. Wickenheiser, I.K. Lednev, Differentiation of hair using ATR FT-IR spectroscopy: a statistical classification of dyed and non-dyed hairs. Forensic Chem. 6, 1-9 (2017). https:// doi.org/10.1016/j.forc.2017.08.001

6. C. Barba, S. Méndez, M. Martí, J.L. Parra, L. Coderch, Water content of hair and nails. Thermochim. Acta 494, 136-140 (2009) 
7. C. Barba, M. Martí, A.M. Manich, J. Carilla, L. Coderch, Water absorption/desorption of human hair and nails. Thermochim. Acta 503-504, 33-39 (2010)

8. M. Egawa, M. Hagihara, M. Yanai, Near-infrared imaging of water in human hair. Skin Res Technol. 19, 35-41 (2013). https://doi.org/10.1111/j.1600-0846.2012.00651.x. (Epub 2012 Jun 4)

9. K. Martin, In vivo measurements of water in skin by near-infrared reflectance. Appl. Spectrosc. 52, 1001-1007 (1998)

10. C.M. Pande, B. Yang, Near-infrared spectroscopy: applications in hair research. J. Soc. Cosmet. Chem. 51, 183-192 (2000)

11. R.E. Imhof, D.J.S. Birch, F.R. Thornley, J.R. Gilchrist, T.A. Strivens, Opto-thermal transient emission radiometry. J. Phys. E Sci. Instrum. 17, 521-525 (1984)

12. P. Xiao, Photothermal radiometry for skin research. Cosmetics 3, 10 (2016). https://doi.org/10.3390/ cosmetics3010010

13. P. Xiao, R.E. Imhof, in Skin Bioengineering Techniques and Applications in Dermatology and Cosmetology, ed. by P. Elsner, A.O. Barel, E. Berardesca, B. Gabard, J. Serup. Current Problems in Dermatology, vol 26 (Karger, Basel, 1998), pp. 48-60. https://doi.org/10.1159/000060575

14. P. Xiao, J.A. Cowen, R.E. Imhof, In-vivo transdermal drug diffusion depth profiling — a new approach to opto-thermal signal analysis. Anal. Sci. 17, 349-352 (2001). https://doi.org/10.14891/analscisp. 17icpp.0.s349.0

15. P. Xiao, Y. Cui, L.I. Ciortea, E.P. Berg, R.E. Imhof, Hair and nail water holding capability measurements by using condenser-TEWL method. Int. J. Cosmet. Sci. 34, 12-16 (2012)

16. R.E. Imhof, E.P. Berg, R.P. Chilcott, L.I. Ciortea, F.C. Pascut, P. Xiao, New instrument for measuremnet water vapour flux density from arbitrary surfaces. IFSCC Mag. 5, 297-301 (2002)

17. R.E. Imhof, M.E.P. de Jesus, P. Xiao, L.I. Ciortea, E.P. Berg, Closed-chamber transepidermal water loss measurement: microclimate, calibration and performance. Int. J. Cosmet. Sci. 31, 97-118 (2009) 